Pathologe 2018 · 39:491

https://doi.org/10.1007/s00292-018-0533-8

Online publiziert: 25 . September 2018

(c) Springer Medizin Verlag GmbH, ein Teil von Springer Nature 2018

CrossMark

\section{W. Roth}

Institut für Pathologie, Universitätsmedizin, Johannes Gutenberg-Universität Mainz, Mainz, Deutschland

\section{Immunpathologie}

Wir erleben momentan einen grundlegenden Umbruch in der Krebstherapie, der im Wesentlichen auf den Erfolgen neuer Immuntherapien und innovativer zielgerichteter Behandlungsansätze beruht. Gerade die Krebsimmuntherapien zeigen bei diversen Tumorarten Behandlungserfolge, die noch vor wenigen Jahren undenkbar schienen, wie zum Beispiel die Langzeit-Remissionsraten beim malignen Melanom. Andererseits stehen wir mit der raschen Weiterentwicklung von Checkpoint-Inhibitoren, zellulären Immuntherapieansätzen und Vakzinierungsstrategien erst am Anfang einer neuen Ära der immunologischen Therapieformen. Obwohl einige wenige Biomarker, wie z. B. PD-L1, bereits in der klinischen Routine angewendet werden, besteht hinsichtlich der Identifizierung und Validierung zuverlässiger prädiktiver Biomarker für die neuen Immuntherapien noch erheblicher Bedarf. Diese rasanten Veränderungen in Diagnostik und Therapie stellen die Pathologie vor beträchtliche Herausforderungen.

Im Rahmen dieses Themenheftes möchten wir die unterschiedlichen Bereiche beleuchten, in denen die neuen Immuntherapieansätze die alltägliche praktische Arbeit der Pathologie verändern. Während die Immunpathologie für lange Zeit ein Schattendasein in der Pathologie fristete, wird gegenwärtig ein grundlegendes Verständnis der Immunologie auch für den Allgemeinpathologen immer wichtiger. Zu diesem Zweck stellen T. Bopp und H. J. Schild in einem Übersichtsartikel zunächst dar, was man als Pathologe von der Immunologie verstehen muss. Das beste Beispiel, dass die tumorimmunpathologische Diagnostik bereits in unserer Routinediagnostik angekommen ist, stellt die PD-L1-Bestimmung dar. Der Artikel von $H$. U. Schildhaus ist eine aktuelle und umfassende Darstellung der PD-L1-Diagnostik, die sich hervorragend als „desk top reference“ für die alltägliche Arbeit eignen wird. Ein Gebiet, dessen Einzug in die Alltagsdiagnostik vermutlich nicht lange auf sich warten lassen wird, ist die Beurteilung von Immunzellinfiltraten für prognostische und/oder prädiktive Zwecke. Dies wird exemplarisch von C. Denkert und Mitarbeitern am Beispiel des Mammakarzinoms dargestellt, während der Artikel von D. C. Wagner perspektivisch einen Überblick über die Bedeutung der Immuninfiltrate und des Tumor-Immun-Microenvironment über alle Tumorentitäten hinweg gibt. Inwiefern die morphologische Erfassung des komplexen Tumor-ImmunMicroenvironments überhaupt noch mit konventionell-lichtmikroskopischer Diagnostik zu leisten ist und ob dies nicht in absehbarer Zeit eine Domäne der digitalen Bildanalyse werden wird, beleuchtet der Artikel von N. Grabe und S. Försch. Der überaus dynamische, spannende und äußerst zukunftsträchtige Grenzbereich zwischen Immunonkologie und Molekularpathologie wird im Artikel von W. Weichert dargestellt.

Die relevanten und spannenden Entwicklungen in der Immunpathologie beschränken sich jedoch nicht nur auf die prädiktive Diagnostik und Therapie von Krebserkrankungen. Ein übergeordnetes und für die allägliche Diagnostik zunehmend wichtiger werdendes Gebiet ist die Pathologie der Medikamentennebenwirkungen, nicht zuletzt weil neue Wirkstoffe mit innovativen Wirkmechanismen in immer kürzeren Abständen in die klinische Patientenversorgung gelangen. $\mathrm{Zu}$ dieser Thematik stellen ausgewiesene Experten die Morphologie von medikamentösen Nebenwirkungen in Leber (B. Straub und Mitarbeiter), Haut (D. Niebel und J. Wenzel), Gastrointestinaltrakt (H. Bläker) und Niere (K. Amann und Mitarbeiter) dar, die in der alläglichen Pathologieroutine mit zunehmender Häufigkeit auftreten.

Prof. Dr. W. Roth

\section{Korrespondenzadresse}

Prof. Dr. W. Roth

Institut für Pathologie, Universitätsmedizin, Johannes Gutenberg-Universität Mainz Langenbeckstraße 1, 55131 Mainz,

Deutschland

wilfried.roth@unimedizin-mainz.de

Interessenkonflikt. W. Roth gibt an, dass kein Interessenkonflikt besteht. 